\title{
Reducing Power of Urine in Lactating Mothers and its Relation to Arakawa's Reaction.
}

\author{
Part 2. \\ 166th Report of the Peroxidase Reaction. \\ (II3th Human Milk Study.) \\ By \\ Kyoshiro Shoda. \\ (正田享四郎) \\ (From the Department of Pediatrics, Faculty of Medicine, \\ Tohoku Imperial University, Sendai. \\ Director: Prof. A. Sato.) \\ Introduction.
}

In Part $1^{1)}$ (the 165 th Report of the Peroxidase Reaction) I reported the relation between thecopper reducing power of lactating mothers' urine and their A rakawa's reaction.

Now, in the present paper I want to report the relation between the permanganate reducing power of lactating mothers' urine and their Arakawa's reaction.

\section{Method of Experiment.}

Method: To 1 c.c. of urine specimen add 2 c.c. of $50 \%$ sulphuric acid and dilute with 10 c.c. of distilled water. Then titrate with 0.04 $\mathrm{N}$ solution of potassium permanganate at the rate of about 0.3 c.c. in one second until the red brown colour of potassium permanganate will not fade instantly with boiling and stirring.

Materials: The urine of mothers of sick and healthy babies who visited the Dispensary of our Department and the Children's Department of the Red Cross Branch Office at Sendai. The urine was obtained between 11 and $12 \mathrm{a} . \mathrm{m}$.

1) Ky. S h od a, Tohoku J. Exp. Med., 1942, 44, 352. 
Arakawa-positive ćases mean cases with milk strongly or normally positive to Arakawa's reaction, and Arakawa-negative cases mean cases with milk completely or almost completely negative to Arakawa's reaction. Weakly Arakawa-positive cases (or intermediate cases) mean cases with milk of intermediate reactions.

\section{Result of Experiment.}

The result is shown in Tables 1,2 and 3. As will be seen from these tables, the permanganate reducing power in 1c.c. of lactating mothers' urine is subject to a fluctuation from 8.5 c.c. to 0.6 c.c. $(0.04 \mathrm{~N}$ potassium permanganate solution).

TABLE 1.

Arakawa-positive cases.

\begin{tabular}{|c|c|c|c|c|c|c|c|}
\hline $\begin{array}{l}\text { No. of } \\
\text { exp. }\end{array}$ & $\begin{array}{l}\text { Date of } \\
\text { exp. }\end{array}$ & $\begin{array}{c}\text { Name } \\
\text { of } \\
\text { infant }\end{array}$ & $\begin{array}{l}\text { Age of } \\
\text { infant } \\
\text { (months) }\end{array}$ & $\begin{array}{l}\text { Age of } \\
\text { mother } \\
\text { (years) }\end{array}$ & $\begin{array}{c}\text { Arakawa's } \\
\text { reaction }\end{array}$ & $\begin{array}{c}\text { Consump- } \\
\text { tion of } \\
\mathrm{KMnO}_{4} \\
\text { solution } \\
\text { (c.c.) }\end{array}$ & $\begin{array}{l}\text { Specific } \\
\text { gravity of } \\
\text { urine }\end{array}$ \\
\hline 1 & 18. $\mathrm{X}$ & M.S. & $13 \mathrm{~m}$ & $32 y$ & $\begin{array}{ll}\mathbf{R} & \mathbf{1}(\mathrm{H}) \\
\mathrm{L} & 4(\mathrm{H}) \\
\end{array}$ & 4.5 & 1030 \\
\hline 2 & 20. & E.M. & $11 \mathrm{~m}$ & $34 \mathrm{y}$ & $\begin{array}{ll}\mathbf{R} & 2(H) \\
\mathrm{L} & 2(H) \\
\end{array}$ & 3.3 & 1024 \\
\hline 3 & 21. XI & H.T. & $9 \mathrm{~m}$ & $29 \mathrm{~g}$ & $\begin{array}{ll}\mathbf{R} & \mathbf{4}(H) \\
\mathrm{L} & 4(H)\end{array}$ & 5.5 & 1030 \\
\hline 4 & 5. XII & A.A. & $7 \mathrm{~m}$ & $35 y$ & $\begin{array}{ll}\mathrm{K} & \mathbf{l}(\mathrm{H}) \\
\mathrm{L} & \mathrm{l}(\mathrm{H})\end{array}$ & 4.1 & 1022 \\
\hline 5 & 6. & K.F. & $10 \mathrm{~m}$ & $25 y$ & $\begin{array}{ll}\mathbf{R} & \mathbf{5}(\boldsymbol{H}) \\
\mathbf{L} & \mathbf{2}(\mathrm{H})\end{array}$ & 4.8 & 1024 \\
\hline 6 & 18. & E.A. & $22 \mathrm{~m}$ & $25 y$ & $\begin{array}{ll}\mathrm{R} & 4(\mathrm{H}) \\
\mathrm{L} & 3(\mathrm{H}) \\
\end{array}$ & 3.5 & 1030 \\
\hline 7 & 15. I & O.S. & $4 \mathrm{~m}$ & $36 \mathrm{y}$ & $\begin{array}{ll}\mathbf{R} & 5(+) \\
\mathrm{L} & \mathbf{1}(\mathrm{H}) \\
\end{array}$ & 2.8 & 1021 \\
\hline 8 & 24. & Y.A. & $10 \mathrm{~m}$ & $38 \mathrm{y}$ & $\begin{array}{ll}\mathbf{R} & \mathbf{1}(H) \\
\mathrm{L} & \mathbf{2 ( H )} \\
\end{array}$ & 3.3 & 1020 \\
\hline 9 & 29. $n$ & K.S. & $3 \mathrm{~m}$ & $29 y$ & $\begin{array}{ll}\mathbf{R} & 1(t) \\
\mathbf{L} & 1(t+)\end{array}$ & 2.2 & 1019 \\
\hline 10 & 3. II & K.MI. & $11 \mathrm{~m}$ & $29 y$ & $\begin{array}{ll}\mathbf{R} & \mathbf{2}(\mathrm{H}) \\
\mathbf{L} & \mathbf{2}(\mathrm{H})\end{array}$ & 4.5 & 1027 \\
\hline 11 & 5, , & Y.H. & $12 \mathrm{~m}$ & $26 \mathrm{y}$ & $\begin{array}{ll}\mathrm{R} & 1(\mathrm{H}) \\
\mathrm{L} & 1(\mathrm{H})\end{array}$ & 6.7 & 1028 \\
\hline 12 & 7. $n$ & K.Y. & $2 \mathrm{~m}$ & $24 y$ & $\begin{array}{ll}\mathbf{R} & 3(H) \\
\mathbf{L} & 3(H) \\
\end{array}$ & 2.8 & 1020 \\
\hline 13 & 9. & K.S. & $6 \mathrm{~m}$ & $31 \mathrm{y}$ & $\begin{array}{ll}\mathbf{R} & 1(H) \\
\mathbf{L} & \mathbf{5}(\mathrm{H}) \\
\end{array}$ & 1.8 & 1014 \\
\hline 14 & 9. n & K.Y. & $9 \mathrm{~m}$ & $32 \mathrm{y}$ & $\begin{array}{ll}\mathbf{R} & \mathbf{1}(\mathrm{H}) \\
\mathbf{L} & \mathbf{2}(\mathrm{H})\end{array}$ & 2.1 & 1015 \\
\hline
\end{tabular}

Table of signs for showing different Arak awa's reaction.

$$
1^{\prime} \quad 2^{\prime} \quad 3^{\prime} \quad 4^{\prime} \quad 5^{\prime}
$$

1. $1(\mathrm{~m})$ stands for $\#$ \# H $\mathrm{H}$ H

2. $1(H), H$ H $\#$ H

3. $2(H), H+H H H$
25. $8( \pm)$ stands for $\pm \pm+\#$ H

26. $9( \pm) \quad n \quad \pm \pm+H+H$

27. $10( \pm) \quad, \pm \pm++H$ 


\begin{tabular}{|c|c|c|c|}
\hline & & & $\begin{array}{lllll}1^{\prime} & 2^{\prime \prime} & 3^{\prime} & 4^{\prime} & 5^{\prime}\end{array}$ \\
\hline 4. & $3(H)$ & $\pi$ & $\mathrm{H} H \mathrm{H} H \mathrm{H}$ \\
\hline 5. & $4(++)$ & $"$ & $\mathrm{H} H \mathrm{H} H \mathrm{H}$ \\
\hline 6. & $5(H)$ & $n$ & $\mathrm{HH} H+H$ \\
\hline 7. & $1(+)$ & $n$ & + + $\#$ \# H \\
\hline 8. & $2(+)$ & n & $+H$ H H H \\
\hline 9. & $3(+)$ & $n$ & $+\#+\#$ H \\
\hline 10. & $4(+)$ & $\pi$ & $+H \mathrm{H} H+$ \\
\hline 11. & $5(+)$ & $n$ & $++\mathrm{H} H \mathrm{H}$ \\
\hline 12. & $6(+)$ & " & $++H H H$ \\
\hline 13. & $7(+)$ & " & $++H+H$ \\
\hline 14. & $8(+)$ & $\eta$ & $+t+H H$ \\
\hline 15. & $9(+)$ & 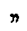 & $+t+H+H$ \\
\hline 16. & $10(+)$ & $n$ & $t+t+H$ \\
\hline 17. & $11(+)$ & $n$ & $+++t+$ \\
\hline 18. & $1( \pm)$ & $n$ & $\pm+H$ H H \\
\hline 19. & $2( \pm)$ & " & $\pm+H+t$ \\
\hline 20. & $3( \pm)$ & 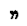 & $\pm+H H+H$ \\
\hline 21. & $4( \pm)$ & $\pi$ & $\pm++H+H$ \\
\hline 22. & $5( \pm)$ & 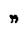 & $\pm++H$ \\
\hline 23. & $6( \pm)$ & $\eta$ & \pm \\
\hline 24. & $7( \pm)$ & 》 & \pm+++ \\
\hline
\end{tabular}

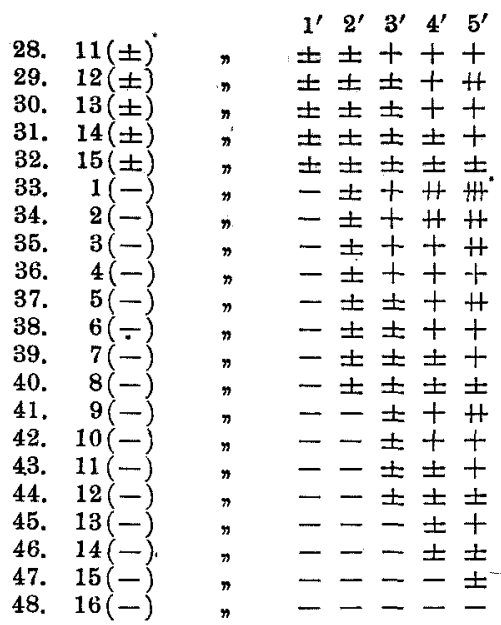

Explanation to the Table:-

Take, for instance, the sign: 2(H). This stands for A rakawa's reaction with the course $(H) 1^{\prime}(H) 2^{\prime}(\mathrm{H}) 3^{\prime}(H) 4^{\prime}(\mathrm{H}) 5^{\prime}$. The sign does not express any prompt result of the reaction, so the prompt reaction of the sign: $2(H)$ may be $(-) 0^{\prime},( \pm) 0^{\prime}$, $(+) 0^{\prime}$ or even $(H) 0^{\prime}$, but this will not matter much, as the result of the reaction in one minute is the most important. Normal reaction is : $(H+1) 1^{\prime}(H) H^{\prime}(H) 3^{\prime}(H) 4^{\prime}(H) 5^{\prime}$.

TABLE 2.

Arakawa-negative cases.

\begin{tabular}{|c|c|c|c|c|c|c|c|}
\hline $\begin{array}{l}\text { No. of } \\
\text { exp. }\end{array}$ & $\begin{array}{c}\text { Date of } \\
\text { exp. }\end{array}$ & $\begin{array}{c}\text { Name } \\
\text { of } \\
\text { infant }\end{array}$ & $\begin{array}{l}\text { Age of } \\
\text { infant } \\
\text { (months) }\end{array}$ & $\begin{array}{l}\text { Age of } \\
\text { mother } \\
\text { (years) }\end{array}$ & $\begin{array}{c}\text { Arakawa's } \\
\text { reaction }\end{array}$ & $\begin{array}{c}\text { Consump- } \\
\text { tion of } \\
\text { KMnO } \\
\text { solution } \\
\text { (c.c.) }\end{array}$ & $\begin{array}{l}\text { Specific } \\
\text { gravity of } \\
\text { urine }\end{array}$ \\
\hline 1 & 5. $X$ & H.H. & $1 \mathrm{~m}$ & $30 \mathrm{~g}$ & $\begin{array}{l}16(-) \\
16(-)\end{array}$ & 3.0 & 1011 \\
\hline 2 & 4. XI & M.K. & $2 \mathrm{~m}$ & $29 \mathrm{y}$ & $\begin{array}{l}16(-) \\
12(-)\end{array}$ & 1.9 & 1010 \\
\hline 3 & 6. \# & H.A. & $15 \mathrm{~m}$ & $26 \mathrm{y}$ & $\begin{array}{l}16(-) \\
16(-)\end{array}$ & 5.1 & 1020 \\
\hline 4 & 10." & Y.S. & $4 \mathrm{~m}$ & $30 \mathrm{y}$ & $\begin{array}{ll}16 & (-) \\
16 & (-)\end{array}$ & 1.4 & 1020 \\
\hline 5 & 17. & A.I. & $1 \mathrm{~m}$ & $30 \mathrm{y}$ & $\begin{array}{l}16(-) \\
16(-)\end{array}$ & 1.4 & 1014 \\
\hline 6 & 24. " & Y.O. & $3 \mathrm{~m}$ & $33 y$ & $\begin{array}{l}16(-) \\
14(-)\end{array}$ & 2.7 & 1020 \\
\hline 7 & 30. & T.S. & $4 \mathrm{~m}$ & $38 \mathrm{y}$ & $\begin{array}{l}12(-) \\
14(-)\end{array}$ & 0.6 & 1006 \\
\hline 8 & 30. & K.T. & $1 \mathrm{~m}$ & $21 \mathrm{y}$ & $\begin{array}{l}15(-) \\
12(-)\end{array}$ & 1.4 & 1010 \\
\hline 9 & 5. XII & T.S. & $9 \mathrm{~m}$ & $30 \mathrm{y}$ & $\begin{array}{l}16(-) \\
16(-)\end{array}$ & 4.5 & 1021 \\
\hline 10 & 7. & N.S. & $5 \mathrm{~m}$ & $25 \mathrm{y}$ & $\begin{array}{l}16(-) \\
15(-)\end{array}$ & 3.9 & 1028 \\
\hline 11 & 13. & K.U. & $3 \mathrm{~m}$ & $25 \mathrm{y}$ & $\begin{array}{l}16(-) \\
16(-)\end{array}$ & 1.8 & 1021 \\
\hline 12 & $16 . n$ & A.S. & $4 \cdot \mathrm{m}$ & $30 \mathrm{y}$ & $\begin{array}{l}14(-) \\
16(-)\end{array}$ & 2.1 & 1020 \\
\hline
\end{tabular}


Reducing Power of Urine and A rakawa's Reaction II.

Table 2. (Continued.)

\begin{tabular}{|c|c|c|c|c|c|c|c|}
\hline $\begin{array}{l}\text { No. of } \\
\text { exp. }\end{array}$ & $\begin{array}{c}\text { Date of } \\
\text { exp. }\end{array}$ & $\begin{array}{c}\text { Name } \\
\text { of } \\
\text { infant }\end{array}$ & $\begin{array}{c}\text { Age of } \\
\text { infant } \\
\text { (months) }\end{array}$ & $\begin{array}{l}\text { Age of } \\
\text { mother } \\
\text { (years) }\end{array}$ & $\begin{array}{l}\text { Arakawa's } \\
\text { reaction }\end{array}$ & $\begin{array}{c}\text { Consump- } \\
\text { tion of } \\
\mathrm{KMnO}_{4} \\
\text { solution } \\
\text { (c.c.) }\end{array}$ & $\begin{array}{l}\text { Specific } \\
\text { gravity of } \\
\text { urine }\end{array}$ \\
\hline 13 & 19. & M.S. & $1 \mathrm{~m}$ & $24 y$ & $\begin{array}{l}16(-) \\
16(-) \\
\end{array}$ & 1.5 & 1022 \\
\hline 14 & 19. & M.K. & $17 \mathrm{~m}$ & $40 \mathrm{~g}$ & $\begin{array}{l}16(-) \\
16(-) \\
\end{array}$ & 7.8 & 1036 \\
\hline 15 & 21. & Y.M. & $15 \mathrm{~m}$ & $28 \mathrm{~J}$ & $\begin{array}{l}16(-) \\
16(-)\end{array}$ & 5.6 & 1035 \\
\hline 16 & 21. & T.K. & $5 \mathrm{~m}$ & $31 \mathrm{y}$ & $\begin{array}{l}15(-) \\
14(-) \\
\end{array}$ & 3.6 & 1026 \\
\hline 17 & 23. $n$ & T.A. & $19 \mathrm{~m}$ & $25 y$ & $\begin{array}{l}16(-) \\
16(-)\end{array}$ & 1.9 & 1026 \\
\hline 18 & 8. I & A.K. & $3 \mathrm{~m}$ & $40 \mathrm{y}$ & $\begin{array}{l}14(-) \\
12(-) \\
\end{array}$ & 2.9 & 1020 \\
\hline 19 & 13. & K.K. & $4 \mathrm{~m}$ & $33 \mathrm{y}$ & $\begin{array}{l}16(-) \\
16(-)\end{array}$ & 1.4 & 1028 \\
\hline 20 & 13. & K.M. & $1 \mathrm{~m}$ & $27 y$ & $\begin{array}{l}16(-) \\
16(-)\end{array}$ & 1.2 & 1020 \\
\hline 21 & 17. , & T.K. & $11 \mathrm{~m}$ & $39 \mathrm{y}$ & $\begin{array}{l}14(-) \\
16(-)\end{array}$ & 4.0 & 1033 \\
\hline 22 & 18. & T.N. & $8 \mathrm{~m}$ & $28 \mathrm{y}$ & $\begin{array}{l}16(-) \\
16(-)\end{array}$ & 2.6 & 1024 \\
\hline 23 & 18. & Y.K. & $\mathbf{3} \cdot \mathbf{m}$ & $27 \mathrm{y}$ & $\begin{array}{l}16(-) \\
16(-)\end{array}$ & 2.9 & 1028 \\
\hline 24 & 1. II & N.E. & $4 \mathrm{~m}$ & $28 \mathrm{y}$ & $\begin{array}{l}16(-) \\
16(-)\end{array}$ & 3.2 & 1023 \\
\hline 25 & 7. & T.M. & $20 \mathrm{~m}$ & $27 \mathrm{~J}$ & $\begin{array}{l}14(-) \\
16(-)\end{array}$ & 4.7 & 1026 \\
\hline
\end{tabular}

Table 3.

Weakly Arakawa-positive cases.

\begin{tabular}{|c|c|c|c|c|c|c|c|}
\hline 1 & 4. $\mathrm{X}$ & T.K. & $7 \mathrm{~m}$ & $29 \mathrm{y}$ & $\begin{array}{ll}2 & (+) \\
2 & (+)\end{array}$ & 5.8 & 1025 \\
\hline .2 & 5. & T.T. & $15 \mathrm{~m}$ & $30 \mathrm{y}$ & $\begin{array}{l}1(H) \\
3(-)\end{array}$ & 7.0 & 1025 \\
\hline 3 & 10. & H.M. & $9 \mathrm{~m}$ & $29 \mathrm{y}$ & $\begin{array}{r}5( \pm) \\
14(-)\end{array}$ & 4.9 & 1022 \\
\hline 4 & 11. & T.S. & $8 \mathrm{~m}$ & $30 \mathrm{y}$ & $\begin{array}{l}7( \pm) \\
4(t) \\
\end{array}$ & 1.8 & 1016 \\
\hline 5 & 11. $\pi$ & T.S. & $2 \mathrm{~m}$ & $38 \mathrm{y}$ & $\begin{aligned} & 16(-) \\
& 3( \pm) \\
&\end{aligned}$ & 5.1 & 1020 \\
\hline 6 & 11." & S. $\mathbf{K}$. & $14 \mathrm{~m}$ & $30 \mathrm{~g}$ & $\begin{array}{l}3(+) \\
5( \pm)\end{array}$ & 4.0 & 1021 \\
\hline 7 & 18. " & I. T. & $8 \mathrm{~m}$ & $36 \mathrm{~g}$ & $\begin{array}{l}7( \pm) \\
3(+) \\
\end{array}$ & 6.7 & 1020 \\
\hline 8 & 18. & S. K. & $2.5 \mathrm{~m}$ & $21 \mathrm{y}$ & $\begin{array}{r}7 \\
16\end{array}( \pm)$ & 3.6 & 1010 \\
\hline 9 & 19. & K.N. & $7 \mathrm{~m}$ & $24 \mathrm{~g}$ & $\begin{array}{r}7(-) \\
16(-)\end{array}$ & 4.1 & 1030 \\
\hline 10 & 19. $\pi$ & K.S. & $1.5 \mathrm{~m}$ & $28 \mathrm{~g}$ & $\begin{aligned} 11 & ( \pm) \\
6 & ( \pm)\end{aligned}$ & 5.7 & 1035 \\
\hline 11 & 30.7 & K.M. & $7 \mathrm{~m}$ & $34 y$ & $\begin{array}{l}6( \pm) \\
7 .(+)\end{array}$ & 1.8 & 1020 \\
\hline
\end{tabular}


Table 3. (Continued.)

\begin{tabular}{|c|c|c|c|c|c|c|c|}
\hline $\begin{array}{l}\text { No. of } \\
\text { exp. }\end{array}$ & $\begin{array}{c}\text { Date of } \\
\text { exp. }\end{array}$ & $\begin{array}{c}\text { Name } \\
\text { of } \\
\text { infant }\end{array} \mid$ & $\begin{array}{c}\text { Age of } \\
\text { infant } \\
\text { (months) }\end{array}$ & $\begin{array}{l}\text { Age of } \\
\text { mother } \\
\text { (years) }\end{array}$ & $\begin{array}{l}\text { Arakawa's } \\
\text { reaction }\end{array}$ & $\begin{array}{c}\text { Consump- } \\
\text { tion of } \\
\mathrm{KMnO}_{4} \\
\text { solution } \\
\text { (c.e.) }\end{array}$ & $\begin{array}{l}\text { Specific } \\
\text { gravity of } \\
\text { urine }\end{array}$ \\
\hline 12 & 4. XI & K.K. & $3 \mathrm{~m}$ & $31 \mathrm{~g}$ & $\begin{array}{ll}7 & ( \pm) \\
7( \pm) \\
\end{array}$ & 1.9 & 1015 \\
\hline 13 & 4. XI & K.S. & $13 \mathrm{~m}$ & $30 \mathrm{y}$ & $\begin{array}{l}1(+) \\
4(+) \\
\end{array}$ & 1.1 & 1020 \\
\hline 14 & 8. XI & K.K. & $6 \mathrm{~m}$ & $27 \mathrm{~g}$ & $\begin{array}{l}3(+) \\
3(+) \\
\end{array}$ & 1.9 & 1010 \\
\hline 15 & 10. & S. K. & $1 \mathrm{~m}$ & $24 \mathrm{~J}$ & $\begin{array}{r}1( \pm) \\
16(+) \\
\end{array}$ & 1.7 & 1010 \\
\hline 16 & 16. XI & H.K. & $8 \mathrm{~m}$ & $40 \mathrm{~g}$ & $\begin{array}{l}4(+) \\
3( \pm) \\
\end{array}$ & 3.7 & 1030 \\
\hline 17 & 16. XI & K.S. & $5 \mathrm{~m}$ & $33 \mathrm{~J}$ & $\begin{array}{l}5( \pm) \\
2(+) \\
\end{array}$ & 2.1 & 1020 \\
\hline 18 & 16. & S. U. & $18 \mathrm{~m}$ & $30 \mathrm{y}$ & $\begin{array}{l}4( \pm) \\
1(+) \\
\end{array}$ & 3.3 & 1020 \\
\hline 19 & 17. & M.Y. & $18 \mathrm{~m}$ & $32 \mathrm{~J}$ & $\begin{array}{l}2(+) \\
3( \pm) \\
\end{array}$ & 6.6 & 1030 \\
\hline 20 & 17. & Y.K. & $20 \mathrm{~m}$ & $34 y$ & $\begin{array}{ll}3 & (-) \\
3 & ( \pm) \\
\end{array}$ & 4.0 & 1020 \\
\hline 21 & 17. & T.S. & $3 \mathrm{~m}$ & $26 \mathrm{y}$ & $\begin{array}{l}7(+) \\
4(+) \\
\end{array}$ & 2.8 & 1024 \\
\hline 22 & 17. & M.S. & $10 \mathrm{~m}$ & $30 y$ & $\begin{array}{l}14(-) \\
11( \pm)\end{array}$ & 2.7 & 1020 \\
\hline 23 & 18. & Y.C. & $17 \mathrm{~m}$ & $37 y$ & $\begin{array}{r}3( \pm) \\
12(-) \\
\end{array}$ & 1.1 & 1015 \\
\hline 24 & 18. & T.S. & $12 \mathrm{~m}$ & $30 y$ & $\overline{2( \pm)}$ & 5.6 & 1020 \\
\hline 25 & 18. & K.I. & $10 \mathrm{~m}$ & $29 \mathrm{y}$ & $\begin{array}{l}9(+) \\
6(-)\end{array}$ & 4.2 & 1020 \\
\hline 26 & 24." & K.S. & $5 \mathrm{~m}$ & $29 \mathrm{~J}$ & $\begin{array}{l}2(+) \\
5( \pm) \\
\end{array}$ & 8.5 & 1080 \\
\hline 27 & 28. & K.I. & $5 \mathrm{~m}$ & $43 \mathrm{y}$ & $\begin{array}{l}2(+4) \\
3(-)\end{array}$ & 4.6 & 1020 \\
\hline 28 & 2. XII & K.T. & $6 \mathrm{~m}$ & 215 & $\begin{array}{l}16(-) \\
13(-) \\
\end{array}$ & 4.0 & 1012 \\
\hline 29 & 4. $n$ & M.B. & $7 \mathrm{~m}$ & $30, y$ & $\begin{array}{r}5( \pm) \\
16(-) \\
\end{array}$ & 4.7 & 1010 \\
\hline 30 & 5. & M.T. & $15 \mathrm{~m}$ & $26 \mathrm{y}$ & $\begin{array}{ll}9 & (+) \\
5 & ( \pm) \\
\end{array}$ & 7.4 & 1036 \\
\hline 31 & 5. $\pi$ & K.o. & $10 \mathrm{~m}$ & $24 \mathrm{y}$ & $\begin{array}{l}7( \pm) \\
6(-) \\
\end{array}$ & 4.4 & 1028 \\
\hline 32 & 12." & S. Y. & $2 \mathrm{~m}$ & $25 \mathrm{y}$ & $\begin{array}{r}16(-) \\
3(-) \\
\end{array}$ & 0.9 & 1015 \\
\hline 33 & 14. ” & K.S. & $2.5 \mathrm{~m}$ & $44 y$ & $\begin{array}{l}5( \pm) \\
5( \pm) \\
\end{array}$ & 2.0 & 1010 \\
\hline 34 & 15." & Y.K. & $10 \mathrm{~m}$ & $25 \mathrm{y}$ & $\begin{array}{l}2(-) \\
5( \pm)\end{array}$ & 2.9 & 1022 \\
\hline 35 & 16." & K.K. & $3 \mathrm{~m}$ & $28 y$ & $\begin{array}{r}2(-) \\
10(-) \\
\end{array}$ & 4.2 & 1027 \\
\hline 36 & 16. " & T.T. & $4 \mathrm{~m}$ & 29 y & $\begin{array}{r}7(-) \\
10(+)\end{array}$ & 2.8 & 1019 \\
\hline
\end{tabular}


Table 3. (Continued.)

\begin{tabular}{|c|c|c|c|c|c|c|c|}
\hline $\begin{array}{l}\text { No. of } \\
\text { exp. }\end{array}$ & $\begin{array}{c}\text { Date of } \\
\text { exp. }\end{array}$ & $\begin{array}{c}\text { Name } \\
\text { of } \\
\text { infant }\end{array}$ & $\begin{array}{c}\text { Age of } \\
\text { infant } \\
\text { (months) }\end{array}$ & $\begin{array}{l}\text { Age of } \\
\text { mother } \\
\text { (years) }\end{array}$ & $\begin{array}{l}\text { Arakawa's } \\
\text { reaction }\end{array}$ & $\begin{array}{c}\text { Consump- } \\
\text { tion of } \\
\mathrm{KMnO}_{4} \\
\text { solution } \\
\text { (c.c.) }\end{array}$ & $\begin{array}{l}\text { Specific } \\
\text { gravity of } \\
\text { urine }\end{array}$ \\
\hline 37 & 18. & S. S. & $6 \mathrm{~m}$ & $31 \mathrm{y}$ & $\begin{array}{l}2(+) \\
9(-)\end{array}$ & 3.9 & 1025 \\
\hline 38 & 19. " & H.O. & $7 \mathrm{~m}$ & $27 \mathrm{~g}$ & $\begin{array}{l}3(-) \\
2(+)\end{array}$ & 2.0 & 1022 \\
\hline 39 & 20. & K.K. & $4 \mathrm{~m}$ & $23 y$ & $\begin{array}{l}7(-) \\
7( \pm)\end{array}$ & 2.6 & 1022 \\
\hline 40 & 8. I & K.Y. & $1.5 \mathrm{~m}$ & $24 \mathrm{y}$ & $\begin{array}{l}3(-) \\
6(-)\end{array}$ & 2.1 & 1015 \\
\hline 41 & 8. & H.о. & $11 \mathrm{~m}$ & $34 y$ & $\begin{array}{l}4(+) \\
5(H)\end{array}$ & 4.0 & 1030 \\
\hline 42 & 11. & I. A. & $11 \mathrm{~m}$ & $24 y$ & $\begin{array}{r}11(-) \\
3(-)\end{array}$ & 4.5 & 1032 \\
\hline 43 & 17. & S.S. & $2.5 \mathrm{~m}$ & $28 \mathrm{y}$ & $\begin{array}{l}5( \pm) \\
5( \pm)\end{array}$ & 1.9 & 1018 \\
\hline 44 & 18. & I. S. & $24 \mathrm{~m}$ & $32 \mathrm{y}$ & $\begin{array}{r}6(-) \\
14(-)\end{array}$ & 3.2 & 1027 \\
\hline 45 & 19 & T.K. & $9 \mathrm{~m}$ & $38 \mathrm{y}$ & $\begin{array}{l}2(+) \\
3( \pm)\end{array}$ & 2.9 & 1019 \\
\hline 46 & 22. $n$ & S.T. & $6 \mathrm{~m}$ & $35 \mathrm{y}$ & $\begin{array}{l}5(+) \\
8(-) \\
\end{array}$ & 2.7 & 1027 \\
\hline 47 & 24. " & E.T. & $11 \mathrm{~m}$ & $26 y$ & $\begin{array}{l}16(-) \\
13( \pm)\end{array}$ & 1.7 & 1028 \\
\hline 48 & 27. & K.N. & $3 \mathrm{~m}$ & $22 y$ & $\begin{array}{r}7(+) \\
7(+) \\
\end{array}$ & 1.7 & 1021 \\
\hline 49 & 27. " & K.W. & $14 \mathrm{~m}$ & $38 y$ & $\begin{array}{l}4(+) \\
4(+) \\
\end{array}$ & 2.2 & 1020 \\
\hline 50 & 29. & H.N. & $1.5 \mathrm{~m}$ & $25 \mathrm{y}$ & $\begin{array}{r}4(-) \\
16(-) \\
\end{array}$ & 3.9 & 1028 \\
\hline 51 & 29. & R.H. & $1.5 \mathrm{~m}$ & $26 \mathrm{y}$ & $\begin{array}{ll}5 & ( \pm) \\
7 & ( \pm)\end{array}$ & 3.6 & 1030 \\
\hline 52 & 1. II & T.T. & $3 \mathrm{~m}$ & $25 \mathrm{y}$ & $\begin{array}{ll}3 & ( \pm) \\
3 & ( \pm) \\
\end{array}$ & 6.7 & 1029 \\
\hline 53 & 1. $\pi$ & Y.S. & $13 \mathrm{~m}$ & $31 \mathrm{y}$ & $\begin{array}{l}2( \pm) \\
2( \pm) \\
\end{array}$ & 4.1 & 1026 \\
\hline 54 & 2. \# & N.Y. & $2 \mathrm{~m}$ & $30 \mathrm{y}$ & $\begin{array}{r}7(-) \\
16(-) \\
\end{array}$ & 1.3 & 1019 \\
\hline 55 & 2. & K.K. & $9 \mathrm{~m}$ & $32 y$ & $\begin{array}{l}5( \pm) \\
2(+) \\
\end{array}$ & 3.2 & 1030 \\
\hline 56 & 3. " & Y.S. & $3 \mathrm{~m}$ & $30 \mathrm{y}$ & $\begin{array}{l}3( \pm) \\
3( \pm) \\
\end{array}$ & 1.7 & 1015 \\
\hline 57 & 6. & T.Y. & $6 \mathrm{~m}$ & $29 \mathrm{y}$ & $\begin{array}{l}7(+) \\
7(+) \\
\end{array}$ & 2.9 & 1023 \\
\hline 58 & 8. & N.0. & $13 \mathrm{~m}$ & $31 \mathrm{y}$ & $\begin{array}{l}7(-) \\
4(+4) \\
\end{array}$ & 6.2 & 1030 \\
\hline 59 & 9. & Y.O. & $2 \mathrm{~m}$ & $26 \mathrm{y}$ & $\begin{aligned} & 12(-) \\
& 3(-) \\
&\end{aligned}$ & 0.6 & 1013 \\
\hline 60 & 9. " & S. K. & $10 \mathrm{~m}$ & 34 y & $\begin{array}{l}6(-) \\
7(+)\end{array}$ & 2.1 & 1020 \\
\hline 61 & 9. " & K.T. & $13 \mathrm{~m}$ & $25 y$ & $\begin{array}{l}7(+) \\
7(+)\end{array}$ & 2.2 & 1022 \\
\hline
\end{tabular}


1. Amount of the consumption of potassium permanganate solution and the intensity of Arakawa's reaction. cases).

a) Arakawa-positive group (including almost Arakawa-positive

Mothers with Arakawa-positive reaction on both sides of breast belonged to this group. The average amount of the consumption of permanganate solution in all the 14 cases was 3.7 c.c. The maximum and the minimum among all the 14 cases were 6.7 c.c. and 1.8 c.c. respectively. The difference of the maximum (6.7) from the minimum (1.8) was $+272 \%$ (Cf. Tables 1 and 4 ).

TABLE 4.

Amount of consumption of potassium permanganate solution and the intensity of A rakawa's reaction.

\begin{tabular}{|c|c|c|c|c|c|c|}
\hline & \multicolumn{2}{|c|}{$\begin{array}{c}\text { Arakawa-positive } \\
\text { case } \\
\text { (14 cases in number) }\end{array}$} & \multicolumn{2}{|c|}{\begin{tabular}{c|} 
Weakly \\
Arakawa-positive case \\
$(61$ cases in number $)$
\end{tabular}} & \multicolumn{2}{|c|}{$\begin{array}{c}\text { Arakawa-negative } \\
\text { case } \\
\text { (25 cases in number) }\end{array}$} \\
\hline & $\begin{array}{c}\text { Consump- } \\
\text { tion of } \\
\text { KMnO }_{4^{-}} \\
\text {solution } \\
\text { (c.c.) }\end{array}$ & $\begin{array}{l}\text { Specific } \\
\text { gravity }\end{array}$ & $\begin{array}{l}\text { Consump- } \\
\text { tion of } \\
\mathrm{KMnO}_{4}- \\
\text { solution } \\
\text { (c.c.) }\end{array}$ & $\begin{array}{c}\text { Specific } \\
\text { gravity } \\
\end{array}$ & $\begin{array}{l}\text { Consump- } \\
\text { tion of } \\
\mathrm{KMnO}_{4^{-}} \\
\text {solution } \\
\text { (c.c.) }\end{array}$ & $\begin{array}{l}\text { Specific } \\
\text { gravity }\end{array}$ \\
\hline Maximum & $\begin{array}{c}6.7 \\
(5.5)^{*}\end{array}$ & $\left.\begin{array}{cc}1 & 030 \\
(1 & 030\end{array}\right)$ & $\begin{array}{c}8.5 \\
(7.4)\end{array}$ & $\begin{array}{cc}1 & 036 \\
\left(\begin{array}{ll}1 & 035\end{array}\right)\end{array}$ & $\begin{array}{c}7.8 \\
(5.6)\end{array}$ & $\begin{array}{c}1036 \\
\left(\begin{array}{ll}1 & 035\end{array}\right)\end{array}$ \\
\hline Minimum & $\begin{array}{c}1.8 \\
(2.1)^{*}\end{array}$ & $\left.\begin{array}{cc}1 & 014 \\
(1 & 015\end{array}\right)$ & $\begin{array}{c}0.6 \\
(0.9)\end{array}$ & $\begin{array}{cc}1 & 010 \\
\left(\begin{array}{ll}1 & 010\end{array}\right)\end{array}$ & $\begin{array}{c}0.6 \\
(1.2)\end{array}$ & $\begin{array}{c}1006 \\
(1010)\end{array}$ \\
\hline Average & 3.7 & 1023 & 3.5 & 1022 & 2.9 & 1022 \\
\hline $\begin{array}{l}\text { Deviation from } \\
\text { minimum to } \\
\text { maximum }\end{array}$ & $+272 \%$ & $+1.6 \%$ & $+1316 \%$ & $+2.6 \%$ & $+12000 \%$ & $+3 \%$ \\
\hline
\end{tabular}

* The data of next maximum and next minimum are put in parenthesis.

b) Arakawa-negative group.

Mothers with completely or almost completely Arakawa-negative reaction on both sides of breast belonged to this group. The average amount of the consumption of permanganate solution in all the 25 cases was 2.9 c.c. The maximum and the minimum among all the 25 cases were 7.8 c.c. and 0.6 c.c. respectively. The difference of the maximum (7.8) from the minimum (0.6) was $+1200 \%$ (Cf. Tables 2 and 4 ).

From these figures one can see that in Arakawa-negative cases the average amount was smaller than in Arakawa-positive cases. And the deviation of the maximum from the minimum was about 5 times larger than in Arakawa-positive cases. 
c) Weakly Arakawa-positive group.

Mothers with intermediate A raka w a's reaction belonged to this group. The average amount of the consumption of permanganate solution in all the 61 cases was 3.5 c.c. The maximum and the minimum among all the 61 cases were 8.5 c.c. and 0.6 c.c. respectively. The difference of the maximum (8.5) from the minimum (0.6) was $+1316 \%$.

From these figures one can see that in this group the average amount comes between the Arakawa-positive and Arakawa-negative groups. And the deviation of the maximum from the minimum is 5 times larger than that of Arakawa-positive group. This attitude of the cases with intermediate reactions-may be said to come near Arakawapositive cases in the average value, while in the deviation they are similar to Arakawa-negative cases.

2. Distribution of all the cases according to the amount of consumption of potassium permanganate solution and the Arakawareaction.

As above described, the average amount of the consumption of permanganate solution needed to oxidize the urine of lactating mothers milk of Arakawa-positive reaction was larger than in the ease of those with milk of the weaker reactions.

However, if individual figures are distributed according to the amount of consumption of permanganate solution and the intensity of the Arakawa reaction, $79 \%$ of Arakawa-positive cases rank between 5.0 c.c. and 2.1 c.c., while only $48 \%$ of Arakawa-negative cases rank in that same range. On the contrary, $52 \%$ of Arakawa-negative cases rank above 5.1 c.c. and below 2.0 c.c., while in the positive cases only $21 \%$ rank there. In the weakly Arakawa-positive cases the relation is intermediate between both extremities as will be seen from Tables 5, 6 and 7. In Arakawa-positive cases the amounts of consumption of permanganate solution fluctuate in a more narrow range than in cases with the weaker reactions.

3. The consumption of permanganate solution in urine of lactating mothers at different periods of lactation.

As shown in Table 8, the average amount of consumption of permanganate solution is smaller in the periods of $1-4$ months after delivery than in the periods of 7-13 months after delivery. As regards the cases over 14 months after delivery they are too few to say anything definite of them. 
TABLE 5 .

Distribution of Arakawa-positive cases according to the amount of potassium permanganate solution and the specific gravity of urine.

\begin{tabular}{|c|c|c|c|c|c|c|c|c|}
\hline \multirow{2}{*}{$\begin{array}{c}\text { Consumption of } \\
\mathrm{KMnO}_{4} \\
\text { solution }\end{array}$} & \multicolumn{8}{|c|}{ Specific gravity } \\
\hline & $\begin{array}{l}\text { Above } \\
1031\end{array}$ & $\begin{array}{l}1030 \\
11 \\
1026\end{array}$ & $\begin{array}{l}1025 \\
1 \\
1021\end{array}$ & $\begin{array}{l}1020 \\
1016\end{array}$ & $\begin{array}{l}1015 \\
1011 \\
1011\end{array}$ & $\begin{array}{r}\text { Below } \\
1010\end{array}$ & \multicolumn{2}{|c|}{ Total } \\
\hline \multicolumn{9}{|l|}{$\begin{array}{c}\text { Above } \\
7.1\end{array}$} \\
\hline $\begin{array}{c}7.0 \\
6.1\end{array}$ & & 1 & & & & & 1 & \multirow[t]{2}{*}{$14 \%$} \\
\hline $\begin{array}{l}6.0 \\
1 \\
5.1\end{array}$ & & 1 & & & & & & \\
\hline $\begin{array}{l}5.0 \\
i \\
4.1\end{array}$ & & 2 & 2 & & $=$ & & 4 & \multirow{3}{*}{$79 \%$} \\
\hline $\begin{array}{l}4.0 \\
3.1 \\
3.1\end{array}$ & & 1 & 1 & 1 & & & 3 & \\
\hline $\begin{array}{l}3.0 \\
1 \\
2.1\end{array}$ & & & 1 & 2 & 1 & & 4 & \\
\hline $\begin{array}{l}2.0 \\
1.1 \\
1.1\end{array}$ & & & & & 1 & & 1 & \multirow{2}{*}{$7 \%$} \\
\hline $\begin{array}{c}\text { Below } \\
1.0\end{array}$ & & & & & & & & \\
\hline \multirow{2}{*}{ Total } & & 5 & 4 & 3 & 2 & & \multirow{2}{*}{\multicolumn{2}{|c|}{$\begin{array}{l}14 \text { cases } \\
\text { in all }\end{array}$}} \\
\hline & & & & & & & & \\
\hline
\end{tabular}

TABLE 6.

Distribution of Arakawa-negative cases according to the amount of potassium permanganate solution and the specific gravity of urine.

$\left.\begin{array}{c|c|c|c|c|c|c|c}\hline \begin{array}{c}\text { Above } \\ 7.1\end{array} & 1 & & & & & & 1 \\ \hline \begin{array}{c}7.0 \\ 1 \\ 6.1\end{array} & & & & & & & \\ \hline \begin{array}{c}6.0 \\ 5.1\end{array} & 1 & & & 1 & & & 2\end{array}\right\}$


TABLE 7.

Distribution of weakly Arakawa-positive cases according to the amount of potassium permanganate solution and the specific gravity of urine.

\begin{tabular}{|c|c|c|c|c|c|c|c|c|}
\hline \multirow{2}{*}{$\begin{array}{c}\text { Consumption of } \\
\mathrm{KMnO}_{4} \\
\text { solution }\end{array}$} & \multicolumn{8}{|c|}{ Specific gravity } \\
\hline & $\begin{array}{c}\text { Above } \\
1031\end{array}$ & $\begin{array}{l}1030 \\
1026 \\
1026\end{array}$ & $\begin{array}{ll}1 & 025 \\
1 & 1 \\
1 & 021\end{array}$ & $\begin{array}{l}1025 \\
1016 \\
1016\end{array}$ & $\begin{array}{l}1015 \\
1011\end{array}$ & $\begin{array}{c}\text { Below } \\
1010\end{array}$ & \multicolumn{2}{|c|}{ Total } \\
\hline $\begin{array}{c}\text { Above } \\
7.1\end{array}$ & 1 & 1 & & & & & 2 & \multirow{3}{*}{$18 \%$} \\
\hline $\begin{array}{r}7.0 \\
1 \\
6.1 \\
\end{array}$ & & 3 & 1 & 1 & & & 5 & \\
\hline $\begin{array}{l}6.0 \\
1 \\
5.1 \\
\end{array}$ & 1 & & 1 & 2 & & & 4 & \\
\hline $\begin{array}{c}5.0 \\
1 \\
4.1 \\
\end{array}$ & I & 4 & 1 & 2 & & 1 & 9 & \multirow{3}{*}{$56 \%$} \\
\hline $\begin{array}{l}4.0 \\
1 \\
3.1 \\
\end{array}$ & $\cdot$ & 6 & 2 & 2 & 1 & 1 & 12 & \\
\hline $\begin{array}{r}3.0 \\
1 \\
2.1 \\
\end{array}$ & & 1 & 5 & 6 & 1 & & 13 & \\
\hline $\begin{array}{l}2.0 \\
1.1 \\
\end{array}$ & & 1 & 2 & 5 & 3 & 3 & 14 & \multirow[b]{2}{*}{$26 \%$} \\
\hline $\begin{array}{c}\text { Below } \\
1.0\end{array}$ & & & & & 2 & & & \\
\hline \multirow{2}{*}{ Total } & 3 & 16 & 12 & 18 & 7 & 5 & \multirow{2}{*}{\multicolumn{2}{|c|}{$\begin{array}{l}14 \text { cases } \\
\text { in all }\end{array}$}} \\
\hline & & & & & & & & \\
\hline
\end{tabular}

TABLE 8.

Consumption of potassium permanganate solution in urine of mothers at different periods of lactation.

\begin{tabular}{c|c|c|c|c}
\hline $\begin{array}{c}\text { Age of infant } \\
\text { (months) }\end{array}$ & $\begin{array}{c}\text { Maximum } \\
\text { (c.c.) }\end{array}$ & $\begin{array}{c}\text { Minimum } \\
\text { (c.c.) }\end{array}$ & $\begin{array}{c}\text { Average } \\
\text { (c.c.) }\end{array}$ & Case \\
\hline 1 & 3.0 & 1.2 & 1.70 & 6 \\
2 & 5.7 & 0.6 & 2.79 & 10 \\
3 & 6.7 & 1.7 & 2.79 & 14 \\
4 & 3.2 & 0.6 & 2.11 & 8 \\
5 & 8.5 & 2.1 & 4.54 & 5 \\
6 & 4.0 & 1.8 & 2.82 & 6 \\
7 & 5.8 & 1.8 & 3.75 & 6 \\
8 & 6.7 & 1.8 & 3.70 & 4 \\
9 & 5.5 & 2.1 & 3.85 & 6 \\
10 & 4.8 & 2.1 & 3.49 & 7 \\
11 & 4.5 & 1.7 & 3.67 & 6 \\
13 & 6.2 & 1.1 & 3.62 & 5 \\
& & & &
\end{tabular}

4. Variation of the consumption of permanganate solution in urine of mothers according to their age.

One can see from Table 9 no definite inclination to increase or decrease according to the age of mothers. 
TABLE 9.

Consumption of potassium permanganate solution in urine of mothers and their age.

\begin{tabular}{c|c|c|c|c}
\hline $\begin{array}{c}\text { Age of mother } \\
\text { (years) }\end{array}$ & $\begin{array}{c}\text { Maximum } \\
\text { (c.c.) }\end{array}$ & $\begin{array}{c}\text { Minimum } \\
\text { (c.c.) }\end{array}$ & $\begin{array}{c}\text { Average } \\
\text { (c.c.) }\end{array}$ & Case \\
\hline 24 & 4.5 & 1.5 & 3.01 & 7 \\
25 & 6.7 & 0.9 & 3.25 & 10 \\
26 & 7.4 & 0.6 & 3.99 & 7 \\
27 & 4.7 & 1.2 & 2.54 & 5 \\
28 & 5.7 & 1.9 & 3.87 & 6 \\
29 & 8.5 & 1.9 & 4.32 & 10 \\
30 & 7.0 & 1.1 & 3.04 & 15 \\
31 & 6.2 & 1.8 & 3.53 & 6 \\
32 & 6.6 & 2.1 & 3.92 & 5 \\
34 & 4.0 & 1.8 & 3.04 & 5 \\
38 & 5.1 & 0.6 & 2.82 & 5 \\
& & & & \\
\hline
\end{tabular}

5. Lastly, as to the relation between the consumption of permanganate solution and the specific gravity of urine, a certain relation seems to exist generally: The larger the specific gravity, the larger the consumption of permanganate solution is. But as will be seen from Table 4, the specific gravity of urine is in Arakawa-positive, weakly Arakawa-positive and Arakawa-negative cases 1023, 1022 and 1022 respectively, or averagingly almost equal. This needs an explanation. As Tables 4, 5 and 6 show, the average specific gravity is equal, but individual cases show a much larger fluctuation in Arakawa-negative cases than in Arakawa-positive cases.

From this point of view one can recognize that the consumption of a larger amount of permanganate solution in Arakawa-positive cases is not proportionate to the specific gravity.

\section{Literature and Comment.}

There are many references concerning the reducing power of urine (Cf. preceding paper ${ }^{1)}$ of mine), but in most of them the power is expressed in terms of glucose using the copper sulphate solution.

As far as I can trace there is only one reference-that of $\mathrm{He}_{\text {lie }} \mathrm{r}^{2}$ -in which the potassium permanganate method was used as in this paper. His method is as follows:

To 10 c.c. of urine add 10 c.c. of concentrated sulphuric acid and titrate with $0.2 \mathrm{~N}$ solution of potassium permanganate until no rapid fading of red-brown colour occurs. At the same time he determined

2) H. Hel i er, Compt. rend. des seanc. de l'Acad. des Sc., 1899, 129, 58. 
the urea content in the same samples of urine. And the reducing power (P) was shown as in the following formula.

$$
\mathrm{P}=20 \times \frac{\mathrm{n}}{\mathrm{m}}
$$

(n . . Titrating value of $\mathrm{KMnO}_{4}$ solution; m. . . Urea content, grms. per litre).

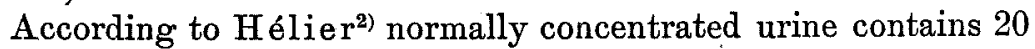
grms. of urea per litre, so in the normally concentrated urine the titrating value of permanganate solution is equal to the reducing power. He further states, the reducing power in healthy adults is very constant (from 12.5 to 15) and in sick adults it shows larger or smaller figures than those in healthy adults. And it is not peculiar to any diseases but. varies according to the course of them, he adds.

In my own result of experiment, the amount of consumption of permanganate solution was in the cases of Arakawa-positive reaction larger than in cases of weaker reactions on an average, but some of Arakawa-negative and weakly Arakawa-positive cases showed a very large or a very small figure compared with those in Arakawa-positive cases. From this view point the majority of mothers with milk of negative or weakly Arakawa-positive reaction may not be healthy in the strict sense of the word if Hélie r's ${ }^{2)}$ result is taken into consideration.

Furthermore, according to Im a $i^{3)}$ (the 186th Report of the Peroxidase Reaction) of our Department, urea content in the urine of mothers with Arakawa-positive reaction is larger than that in Arakawanegative cases. So that if Hélier's ${ }^{2)}$ method be applied to these specimens, the result will become clearer than that in the present paper.

Moreover, as was cited in my preceding paper ${ }^{1)}$, Moor ${ }^{4}$, who used the berlin blue method, is of the opinion that the reducing power in the urine of persons in a state of anemia, neurasthenia or general prostration is very poor compared with that of normal persons. From this view point too, mothers with milk of Arakawa-negative reaction may not be healthy in the strict sense of the word in a majority of cases.

\section{Summary and Conclusions.}

1. The reducing power of urine measured by the consumption of potassium permanganate solution in Arakawa-positive cases are on an

3) S. Imai, Tohokn J. Exp. Med., 186th Report of the Peroxidase Reaction (to be published in this Journal).

4) Wm. O. Moor, Deut. med. Wschr., 1925, 51, 1436. 
average larger than in Arakawa-negative cases. However, the urine in some Arakawa-negative cases consumed a very large and others a very small amount of potassium permanganate solution.

2. The consumption of potassium permanganate solution shows lower figures in early periods of lactation ( $1-4$ months after delivery) than in later periods. But there is no relation between the amount of consumption of potassium permanganate solution and the age of the mother.

3. "Healthy" mothers with Arakawa-negative reaction may be classed unhealthy, at least in a majority of cases, if we take into consideration the result shown by Hélier ${ }^{2}$ and $\mathrm{Moor}^{4}$. 